\title{
Hypothalamic Deep Brain Stimulation in Positron Emission Tomography
}

\author{
Arne May, ${ }^{1}$ Massimo Leone, ${ }^{2}$ Henning Boecker, ${ }^{3}$ Till Sprenger, ${ }^{4}$ Tim Juergens, ${ }^{5}$ Gennaro Bussone, ${ }^{2}$ and \\ Thomas R. Tolle ${ }^{4}$ \\ ${ }^{1}$ Department of Systems Neuroscience, University of Hamburg, D-20246 Hamburg, Germany, ${ }^{2}$ Instituto Neurologico Carlo Besta, 20133 Milano, Italy, \\ Departments of ${ }^{3}$ Nuclear Medicine and ${ }^{4}$ Neurology, Technical University of Munich, 81657 Munich, Germany, and ${ }^{5}$ Department of Neurology, University of \\ Regensburg, 93059 Regensburg, Germany
}

Recently, functional imaging data have underscored the crucial role the hypothalamus plays in cluster headache, one of the most severe forms of primary headache. This prompted the application of hypothalamic deep brain stimulation. Yet, it is not apparent how stimulation of an area that is thought to act as a pace-maker for acute headache attacks is able to prevent these attacks from occurring. We addressed this issue by examining 10 operated chronic cluster headache patients, using $\mathrm{H}_{2}{ }^{15} 0$-positron emission tomography and alternately switching the hypothalamic stimulator on and off. The stimulation induced activation in the ipsilateral hypothalamic gray (the site of the stimulator tip), the ipsilateral thalamus, somatosensory cortex and praecuneus, the anterior cingulate cortex, and the ipsilateral trigeminal nucleus and ganglion. We additionally observed deactivation in the middle temporal gyrus, posterior cingulate cortex, and contralateral anterior insula. Both activation and deactivation are situated in cerebral structures belonging to neuronal circuits usually activated in pain transmission and notably in acute cluster headache attacks. Our data argue against an unspecific antinociceptive effect or pure inhibition of hypothalamic activity. Instead, the data suggest a hitherto unrecognized functional modulation of the pain processing network as the mode of action of hypothalamic deep brain stimulation in cluster headache.

Key words: deep brain stimulation; hypothalamus; cluster headache; PET, pain; neuromodulation

\section{Introduction}

Neuroimaging with positron emission tomography (PET) shed light on the genesis of two of the most important headache syndromes, documenting activation in the midbrain and pons in migraine (Afridi et al., 2005) and in the hypothalamic gray in cluster headache (CH) (May et al., 1998; Sprenger et al., 2004). These areas are not simply involved as a response to first division nociceptive pain impulses but are inherent to each syndrome, probably in some permissive or dysfunctional role (Goadsby, 2002; May, 2005). Furthermore, using high-resolution structural three-dimensional (3D) magnetic resonance images and voxelbased morphometry, a significant structural difference in gray matter density of the hypothalamus was found in patients with $\mathrm{CH}$ when compared with healthy volunteers (May et al., 1999). The colocalization of morphometric and functional changes demonstrate the precise anatomical location for a probable CNS lesion in $\mathrm{CH}$. Given that this area is involved in circadian rhythms, sleep-wake cycling (Moore, 1997), and control of the

Received 0ct. 27, 2005; revised Feb. 9, 2006; accepted Feb. 13, 2006.

A.M. is supported by a grant from the Deutsche Forschungsgemeinschaft (MA 1862/2). H.B. and T.R.T. are supported by Sonderforschungsbereich 391, Teilprojekt 9 , and the Kommission klinische Forschung medizinischer Bereich Technische Universität Projekt 8764153. We thank the radiographers and cyclotron personnel of the Department of Nuclear Medicine at the University of Munich for technical support during the scanning, E. Schoell for editing this manuscript, and our patients who generously helped with this study.

Correspondence should be addressed to Dr. Arne May, Assistant Professor of Neurology, Department of Systems Neuroscience, Universitäts-Krankenhaus Eppendorf, Martinistrasse 52, D-20246 Hamburg, Germany. E-mail: a.may@uke.uni-hamburg.de.

DOI:10.1523/JNEUROSCI.4609-05.2006

Copyright $\odot 2006$ Society for Neuroscience $\quad$ 0270-6474/06/263589-05\$15.00/0 autonomic system (Overeem et al., 2002), these data suggest an involvement of this hypothalamic area as a primum movens in the acute cluster attack. These findings prompted the use of deep brain stimulation (DBS) in the posterior hypothalamic gray matter in a patient with intractable $\mathrm{CH}$ headache and led to a complete relief of attacks (Leone et al., 2001). To date, 20 operated intractable $\mathrm{CH}$ patients have been reported (Franzini et al., 2003; Leone et al., 2006), some with a follow up of $>4$ years (Leone et al., 2004a; Schoenen et al., 2005).

Little is known about the circuits and mechanisms underlying the analgesic effect of DBS; however, it probably involves activation of thalamocortical pathways and changes in cortical activity (Kumar et al., 1997). The uniqueness of the DBS approach is that it allows in vivo investigating of the functional role of the underlying neuronal circuits by switching the hypothalamic stimulator on and off. It is unclear whether DBS causes (1) a local blockade of the hypothalamic trigger activity, (2) a direct antinociceptive effect by activation of the periaqueductal gray (PAG) and/or rostral ventromedial medulla (RVM) or, (3) a modulation of neuronal pain-processing pathways. To unravel the brain circuitry mediating stimulation-induced effects, we applied PET, a relatively noninvasive imaging technique that is sensitive to changes in regional cerebral blood flow ( $\mathrm{rCBF}$ ) as an indirect measure of neuronal activity in humans (Frackowiak and Friston, 1994).

\section{Materials and Methods}

Patients. Ten right-handed patients (nine men and one woman) aged 25-63 years, with hypothalamic DBS electrodes (five left, five right), gave written informed consent to participate in the PET study. The proce- 
Table 1. Demographic data of the 10 patients who underwent PET examination

\begin{tabular}{|c|c|c|c|c|c|c|c|c|c|}
\hline & $\begin{array}{l}\text { Age at } \\
\text { scanning } \\
\text { (years) }\end{array}$ & Sex & $\mathrm{CH}$ since & CH side & Date operated & Date scanned & Pain free? & $\begin{array}{l}\text { Stimulus characteristics } \\
{[\text { amplitude }(V) / \text { pulse width }} \\
(\mu s) / \text { frequency }(\mathrm{Hz})]\end{array}$ & Preventative medication \\
\hline Pat 1 & 41 & M & 1997 & Left & July 2000 & May 2002 & Yes & $3.2 \mathrm{~V} / 60 \mu \mathrm{s} / 180 \mathrm{~Hz}$ & None \\
\hline Pat 2 & 52 & M & 1997 & Left & November 2000 & May 2002 & Yes & $1.1 \mathrm{~V} / 60 \mu \mathrm{s} / 180 \mathrm{~Hz}$ & Methysergide, 4 mg; Verapamil, $240 \mathrm{mg}$ \\
\hline Pat 3 & 64 & $\mathrm{~F}$ & 1994 & Left & May 2001 & May 2002 & Yes & $3.0 \mathrm{~V} / 60 \mu \mathrm{s} / 180 \mathrm{~Hz}$ & Verapamil, $80 \mathrm{mg} / \mathrm{d}$ \\
\hline Pat 4 & 52 & M & 1997 & Right & October 2001 & May 2002 & Yes & $3.1 \mathrm{~V} / 60 \mu \mathrm{s} / 180 \mathrm{~Hz}$ & Methysergide, $4 \mathrm{mg}$ \\
\hline Pat 5 & 30 & M & 2000 & Left & March 2002 & May 2002 & Occasional attacks & $1.4 \mathrm{~V} / 60 \mu \mathrm{s} / 180 \mathrm{~Hz}$ & None \\
\hline Pat 6 & 25 & M & 2002 & Left & October 2003 & November 2004 & Occasional attacks & $3.3 \mathrm{~V} / 60 \mu \mathrm{s} / 180 \mathrm{~Hz}$ & Verapamil, $360 \mathrm{mg}^{a}$ \\
\hline Pat 7 & 51 & M & 2002 & Right & August 2003 & November 2004 & Yes & $2.6 \mathrm{~V} / 60 \mu \mathrm{s} / 180 \mathrm{~Hz}$ & Verapamil, $360 \mathrm{mg}^{b}$ \\
\hline Pat 8 & 51 & M & 2002 & Right & November 2003 & November 2004 & Yes & $1.7 \mathrm{~V} / 60 \mu \mathrm{s} / 180 \mathrm{~Hz}$ & None \\
\hline Pat 9 & 25 & $M$ & 2002 & Right & July 2003 & November 2004 & Yes & $2.1 \mathrm{~V} / 60 \mu \mathrm{s} / 180 \mathrm{~Hz}$ & None \\
\hline Pat 10 & 58 & M & 2000 & Right & May 204 & November 2004 & Yes & $2.5 \mathrm{~V} / 60 \mu \mathrm{s} / 180 \mathrm{~Hz}$ & None \\
\hline
\end{tabular}

All patients suffered from four to eight attacks before the operation. Only patients 5 and 6 still suffered from occasional attacks at the time of scanning. Patient 5 was operated on 1 month before PET (operated end of March, scanned beginning of May) and became attack free 4 weeks later. Pat, Patient; $M$, male; $F$, female.

${ }^{a}$ To prevent $\mathrm{CH}$ attacks contralateral to the stimulation side, which started well before the implantation.

${ }^{b}$ Not for $\mathrm{CH}$ but because of previous myocardial infarction.

dures were approved by the Ethics Committee of the Technische Universität of Munich and the Istituto Neurologico Carlo Besta. The DBS electrodes had been implanted to treat the patients intractable chronic cluster headache, following the guidelines for selecting eligible patients (Leone et al., 2004b). Before the operation, all patients suffered from four to eight attacks per day and were drug refractory to all medications used to treat $\mathrm{CH}$. All patients had undergone stereotactic functional surgery in Milan (Franzini et al., 2003). The surgery had included microelectrode mapping and implantation of hypothalamic electrodes (Medtronic, Minneapolis, MN). The stimulator tip was situated in the posterior inferior hypothalamic gray (May et al., 1998, 1999). Therapeutic stimulation was continuous and unipolar. Eight patients were headache free after stereotactic activation; none experienced any relevant side effects. On the day of scanning, five patients were taking additional preventive medication. Two patients suffered occasionally from acute cluster attacks, one of whom had only recently been operated (patient 5; operated end of March; scanned beginning of May). Table 1 demonstrates demographic data for the 10 patients.

Design. Using a block design and alternately switching the hypothalamic stimulator on and off, each patient underwent 12 consecutive $\mathrm{H}_{2}{ }^{15} \mathrm{O}$-water PET scans during two conditions: (1) baseline and (2) during DBS. Artificial stimulation of brain structures may change brain activation patterns over time. Considering the limited patient population, it was not possible to use different timing strategies (i.e., stimulation intervals). Because the present study focuses on acute effects of hypothalamic stimulation and above all to guarantee comparable conditions, the stimulator was turned on or off exactly $60 \mathrm{~s}$ before the start of each scan and was kept in this position until $60 \mathrm{~s}$ before the consecutive scan (Fig. $1 A)$. Subjects had their eyes closed during all scans.

Data acquisition and analysis. PET scans were performed with an ECAT EXACT HR + scanning system (Siemens, Knoxsville, TN) in 3D mode with septa retracted. A neck shield (NeuroShield; Scanwell Systems, Lavigne, Montreal, Canada) was used to reduce random count rates. An antecubital vein cannula was used to administer the tracer, 10 $\mathrm{mCi}$ of $\mathrm{H}_{2}{ }^{15} \mathrm{O}$ per run. The activity was flushed into subjects as a semibolus over $30 \mathrm{~s}$ at a rate of $14 \mathrm{ml} / \mathrm{min}$. The data were acquired in one $90 \mathrm{~s}$ frame beginning $5 \mathrm{~s}$ before the peak of the head curve. The interval between scans was $10 \mathrm{~min}$. Attenuation correction was performed with a transmission scan acquired at the beginning of each study. Images were reconstructed by filtered back projection into 63 image planes (separation, $2.4 \mathrm{~mm}$ ) and into a $128 \times 128$ pixel image matrix (pixel size, $2.1 \times$ $\left.2.1 \mathrm{~mm}^{2}\right)$. Because cluster headache is a strictly lateralized syndrome (Headache Classification Committee of the International Headache Society, 2004), we mirrored the PET scans in the patients with left-sided headache in the axial plane to be able to analyze all subjects together. SPM2 (Wellcome Department of Imaging Neuroscience, London, UK; http://www.fil.ion.ucl.ac.uk/spm) was used for data analysis. Images were realigned with the first image as the reference and then spatially normalized into standard stereotactic space. The normalized images were smoothed with a Gaussian filter of $10 \mathrm{~mm}$ full width at half maximum. Statistical parametric maps were derived with prespecified contrasts, comparing rCBF during stimulation and rest. Significance levels for the one-tailed $t$ statistics were set at $p<0.001$ uncorrected, based on our strong regional a priori hypothesis based on the clinical and experimental data cited in the text. Based on this a priori hypothesis regarding the involvement of pain transmitting structures, such as the hypothalamus, thalamus, anterior cingulate cortex, and the insular cortex, we applied small volume correction to these areas with a threshold of $p<0.05$ for multiple comparisons.

\section{Results}

All the patients experienced rigorous improvement in headache frequency after hypothalamic stimulation was initiated: eight of the investigated 10 patients were completely pain free and the remaining two suffered only from sporadic attacks. Notably, it takes several days, or even weeks, between turning the unipolar stimulator on or off and observing a change in the clinical picture (Franzini et al., 2003). None of the patients experienced any adverse side effects or headache symptoms during the scanning.

Categorical SPM analysis revealed stimulator-induced activations (on $>$ off) in the ipsilateral posterior inferior hypothalamic gray (the site of the stimulator tip), the ipsilateral thalamus, somatosensory cortex and praecuneus, the anterior cingulate cortex, and the ipsilateral trigeminal nucleus and ganglion. Significant deactivations ( off $>$ on) were found in the middle temporal gyrus, posterior cingulate cortex, inferior temporal gyrus bilaterally, and contralateral anterior insula. Table 2 and Figure $1 B$ demonstrate activation and deactivation in hypothalamic DBS.

\section{Discussion}

Deep brain stimulation allows a highly focal and reversible stimulation of neuronal target structures. Attempts to alleviate medically intractable pain through continuous stimulation of deep brain structures have been reported for nearly half a century (Mazars et al., 1960; Raskin et al., 1987; Bittar et al., 2005). Over the years, several brain regions have been targeted with varying degrees of success. These include the ventroposterolateral nucleus and several other thalamic nuclei, the periaqueductal and periventricular gray matter, and motor cortex (Nandi et al., 2002). Some authors recommend PAG/periventricular gray stimulation for the treatment of peripheral pain (Kumar et al., 1997), whereas stimulation of thalamic sensory relay nuclei ventroposterior lateral nucleus or ventroposterior medial nucleus or the internal capsule has been suggested for the alleviation of cen- 
A)

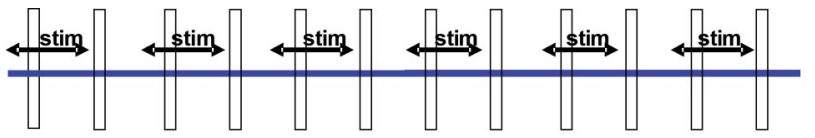

Scan

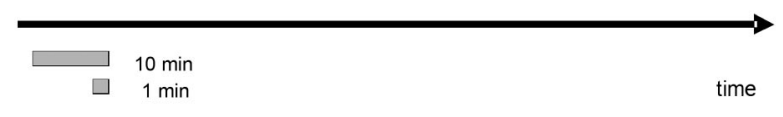

B)

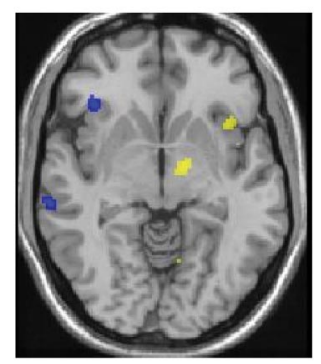

D)

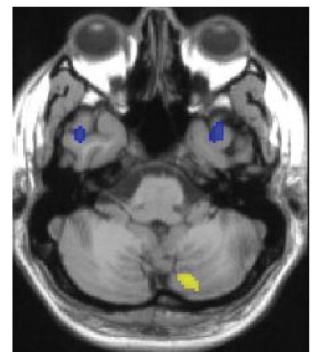

C)

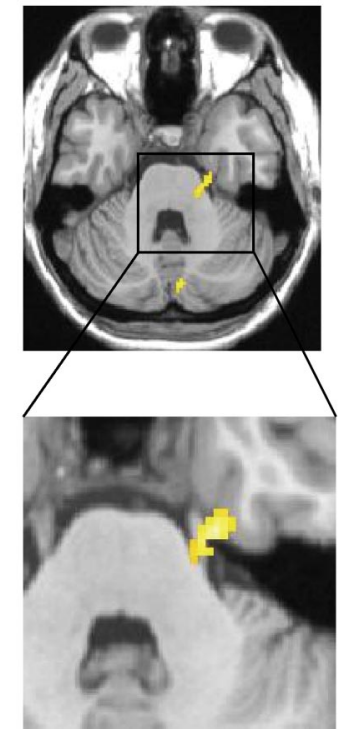

Figure 1. A, Design of the PET study in 10 cluster headache patients. Using a block design and alternately switching the hypothalamic stimulator on and off, each patient had 12 consecutive $\mathrm{H}_{2}{ }^{15} 0$-water PET scans during two conditions: (1) baseline (stimulator off) and (2) during DBS (stimulator on). To allow for comparable conditions, the stimulator was turned on or off exactly $60 \mathrm{~s}$ before the start of each scan and was kept in this position until $60 \mathrm{~s}$ before the consecutive scan. stim, Stimulation. $\boldsymbol{B}-\boldsymbol{E}$, Comparison of hypothalamic stimulator on and off condition in 10 patients with chronic cluster headache. The activations during the condition stimulator on are displayed as statistical parametric maps that show the areas of significant rCBF increases $(p<0.05)$ in yellow superimposed on a normalized T1-weighted image of a healthy control subject. Significant activation was detected in the ipsilateral posterior inferior hypothalamic gray (the site of the stimulator tip) and the ipsilateral insula $(\boldsymbol{B})$ and the ipsilateral trigeminal nucleus and ganglion ( $\boldsymbol{C}$. Additionally significant deactivations during the condition stimulator on are displayed as statistical parametric maps that show the areas of significant rCBF decreases $(p<0.05)$ in blue superimposed on a normalized T1-weighted image of a healthy control subject. Deactivations occurred in the contralateral insula and the primary somatosensory cortex $(\boldsymbol{B})$ and in the inferior temporal cortex bilaterally $(\boldsymbol{D})$. Both activations and deactivations are situated in cerebral structures belonging to neuronal circuits usually activated in pain transmission. $\boldsymbol{E}$ magnifies the same axial view as $\boldsymbol{C}$, to better visualize the finding. The right side of the picture is the right side of the brain.

tral pain (Davis et al., 2000). Although animal experiments suggest the lateral hypothalamus to be involved in pain modulation (Dafny et al., 1996; Workman and Lumb, 1997), serving as a relay station for nociceptive transmission and autonomic function (Randich and Gebhart, 1992), electrical stimulation of the hypothalamus to produce analgesia has only been used in experimental animals (Lopez et al., 1991). Conversely, electrical stimulation of the superior sagittal sinus activates the supra-optic nucleus and posterior hypothalamic area (Benjamin et al., 2004) [a monosynaptic pathway connecting the hypothalamus and trigeminal nucleus has been documented (Malick et al., 2000)]. The posterior hypothalamus is able to both decrease and enhance nociceptive responses in the trigeminal nucleus caudalis (Bartsch et al., 2004). In humans, stereotactic thermocoagulation of the posteromedial hypothalamus has been successfully used to treat otherwise intractable cancer pain (Sano et al., 1975)

Using PET, we investigated 10 patients successfully treated with hypothalamic DBS for intractable $\mathrm{CH}$. Our data shed light on mechanisms by which hypothalamic DBS interferes with brain circuitries. The focus of the present study was to reach a better understanding of the acute effects of hypothalamic stimulation in humans, rather than the influence of deep brain stimulation on alleviating cluster attacks; indeed, all of our patients were headache free during the scanning.

After hypothalamic stimulation, we observed activation (thalamus, somatosensory cortex, praecuneus, anterior cingulate cortex, and trigeminal nucleus and ganglion) and deactivations (middle temporal gyrus, posterior cingulate cortex, and anterior insula) in cerebral structures, which belong to neuronal circuits usually activated in pain transmission and acute $\mathrm{CH}$ attacks.

Based on observations from previous PET studies (May, 2003), it may be hypothesized that the symptoms of $\mathrm{CH}$ are caused by a low threshold "oscillator" that is generated by the hypothalamus and subsequently activates cortical structures of the pain-transmitting system leading to the characteristic shortlived trigemino-autonomic pain. Nevertheless, it was so far obscure how the (deep brain) stimulation of this precise triggering area prevents cluster headache attacks from occurring. Our data, for the first time, provide evidence that DBS of the hypothalamus not only depolarizes this region (i.e., local depolarization and a local flow response to neuronal firing), but also bidirectionally modulates activity in fundamental structures of the ascending pain pathway. The fact that key structures of the descending antinociceptive system (PAG and RVM), although densely interconnected with the hypothalamus (Vertes and Crane, 1996), were not influenced by hypothalamic DBS, essentially excludes a purely antinociceptive mode of action.

It needs to be pointed out that a weakness in our study is that the data can only be analyzed by pooling results from several patients. From a clinical point of view, it is also important to mention that although it may take weeks or even longer before the hypothalamic deep brain stimulation is effective, we investigated hypothalamic deep brain stimulation immediately after turning the stimulator on or off. The application of fluorodeoxyglucose-PET techniques before and after implantation would offer an opportunity to study the long-lasting alterations in metabolic brain activity. An alternative approach to understand the modulatory effects of hypothalamic DBS would be to use an experimental pain paradigm in the on condition or the off condition. However, none of the patients who received hypothalamic deep brain stimulation suffered from trigeminal hypoesthesia or anesthesia, and it is intriguing that hypothalamic stimulation does not affect anesthesia dolorosa (Leone et al., 2004a). Considering the complex pattern of activation in some and deactivation in other pain processing areas distant from the hypothalamus, an exclusive focal block (e.g., depolarization effect of the posterior hypothalamic area as the sole therapeutic effector) is unlikely as well. This observation strengthens the hypothesis that the pain of cluster headache does not arise from a primary dysfunction of the trigeminal nerve itself, but is generated directly from the brain, involving a complex neuronal network.

It is an intriguing finding that turning the stimulator on causes an ipsilateral activation of the trigeminal nucleus and ganglion (Fig. 1) in patients suffering from a syndrome concisely ascribable to the trigeminovascular system and not to somatic or chronic pain as such. Furthermore, the scanning was performed in the absence of any facial or head pain, and none of the operated patients has ever reported any trigeminal negative or positive 
Table 2. Significant increases and decreases in regional blood flow in the patients during the scans defined for the condition stimulator on compared with the condition stimulator off

\begin{tabular}{|c|c|c|c|c|c|c|c|c|c|}
\hline \multirow[b]{3}{*}{ Region } & \multirow[b]{3}{*}{ Brodmann areas } & \multicolumn{4}{|c|}{ Activation in hypothalamic stimulation $(n=10)$} & \multicolumn{4}{|c|}{ Deactivation in hypothalamic stimulation $(n=10)$} \\
\hline & & \multicolumn{3}{|c|}{ Talairach coordinates } & \multirow[b]{2}{*}{ Zscore of peak activation } & \multicolumn{3}{|c|}{ Talairach coordinates } & \multirow[b]{2}{*}{ Zscore of peak deactivation } \\
\hline & & $x$ & $y$ & $z$ & & $x$ & $y$ & $z$ & \\
\hline Precuneus & BA 7 & 4 & -48 & 46 & $Z=4.08$ & & & & \\
\hline Middle temporal gyrus & BA39 & -40 & -86 & 28 & $Z=4.05$ & & & & \\
\hline Hypothalamus $^{a}$ & & 14 & -8 & -8 & $Z=3.67$ & & & & \\
\hline Thalamus $^{a}$ & & 4 & -10 & 8 & $Z=3.88$ & & & & \\
\hline Inferior temporal gyrus & BA20 & 24 & -14 & -36 & $Z=3.83$ & & & & \\
\hline Anterior cingulate cortex ${ }^{a}$ & BA24 & -10 & 24 & -14 & $Z=3.83$ & & & & \\
\hline Posterior cingulate cortex & BA30 & 20 & -54 & 20 & $Z=3.71$ & & & & \\
\hline Primary somatosensory cortex & BA 2 & -54 & -18 & 24 & $Z=3.59$ & & & & \\
\hline Trigeminal nucleus & & 14 & -28 & -28 & $Z=3.48$ & & & & \\
\hline Cerebellum/vermis & & 10 & -54 & -16 & $Z=4.24$ & & & & \\
\hline Anterior insula & & 38 & 16 & -10 & $Z=3.56$ & -38 & 28 & -6 & $Z=3.48$ \\
\hline Middle temporal gyrus & BA21 & & & & & -50 & -50 & 10 & $Z=4.10$ \\
\hline \multirow[t]{2}{*}{ Inferior temporal gyrus } & BA38 & & & & & -46 & 8 & -48 & \\
\hline & & & & & & 34 & 10 & -44 & $\begin{array}{l}Z=4.06 \\
Z=3.44\end{array}$ \\
\hline Superior frontal gyrus & BA10 & & & & & -18 & 58 & 18 & $Z=3.76$ \\
\hline Posterior cingulate cortex & BA24 & & & & & 16 & -10 & 42 & $Z=3.73$ \\
\hline
\end{tabular}

The activation and deactivation are tabulated in terms of the activated brain regions and their Brodmann areas, the $x, y, z$ coordinates to the standardized anatomical space, and refer to the relative distance to the anterior commissureposterior commissure line (joining the anterior and posterior commissures), which is situated at $0 \mathrm{~mm}$. Each location is the peak within a cluster (defined as the voxel with the highest $Z$ score; $p<0.05$ ). L, Left; $r$, right; $B A$, Brodmann area. ${ }^{a}$ Small volume correction.

sensation in association with the stimulator activity. Although this finding could represent a local inhibition of the trigeminal ganglion and nucleus, the clinical impression as well as recent results of sensory testing in $\mathrm{CH}$ patients with hypothalamic DBS (Schoenen et al., 2005) argue for a more complex mechanism.

This work cannot explain why it takes several weeks after implantation of the electrodes and stimulation to terminate cluster attacks and why it takes again several weeks after tuning the stimulator off until cluster attacks reappear. This time frame suggests that the hypothalamus may be best described as a "clock-pulse generator," which must oscillate in a specific manner over time to modulate distant autonomic and trigemino-vascular areas, resulting in unilateral pain and autonomic symptoms (May, 2005). Following this theory, the constant depolarization would discontinue the biological clock-like impulses from the distant trigeminal and autonomic "executers" involving a certain time lag.

From a methodological perspective, one has to point out that the $60 \mathrm{~s}$ pause between the conditions on and off may result in an insufficient "washout" from previous effects and consequently that the effects of DBS over the course of $10 \mathrm{~min}$ may not be representative of the neuronal effects of DBS over weeks. Intuitively, it is very likely that delayed neuronal effects are as important in mechanism of pain relief as the acute effects. The focus of the present study was to better understand the acute effects of hypothalamic stimulation in humans, knowledge important for the future understanding of long-term stimulator effects (i.e., effects occurring after weeks or months of stimulation).

\section{References}

Afridi SK, Matharu MS, Lee L, Kaube H, Friston KJ, Frackowiak RS, Goadsby PJ (2005) A PET study exploring the laterality of brainstem activation in migraine using glyceryl trinitrate. Brain 128:932-939.

Bartsch T, Levy MJ, Knight YE, Goadsby PJ (2004) Differential modulation of nociceptive dural input to [hypocretin] orexin A and B receptor activation in the posterior hypothalamic area. Pain 109:367-378.

Benjamin L, Levy MJ, Lasalandra MP, Knight YE, Akerman S, Classey JD, Goadsby PJ (2004) Hypothalamic activation after stimulation of the superior sagittal sinus in the cat: a Fos study. Neurobiol Dis 16:500-505.

Bittar RG, Kar-Purkayastha I, Owen SL, Bear RE, Green A, Wang S, Aziz TZ
(2005) Deep brain stimulation for pain relief: a meta-analysis. J Clin Neurosci 12:515-519.

Dafny N, Dong WQ, Prieto-Gomez C, Reyes-Vazquez C, Stanford J, Qiao JT (1996) Lateral hypothalamus: site involved in pain modulation. Neuroscience 70:449-460.

Davis KD, Taub E, Duffner F, Lozano AM, Tasker RR, Houle S, Dostrovsky JO (2000) Activation of the anterior cingulate cortex by thalamic stimulation in patients with chronic pain: a positron emission tomography study. J Neurosurg 92:64-69.

Frackowiak RS, Friston KJ (1994) Functional neuroanatomy of the human brain: positron emission tomography-a new neuroanatomical technique. J Anat 184:211-225.

Franzini A, Ferroli P, Leone M, Broggi G (2003) Stimulation of the posterior hypothalamus for treatment of chronic intractable cluster headaches: first reported series. Neurosurgery 52:1095-1101.

Goadsby PJ (2002) Pathophysiology of cluster headache: a trigeminal autonomic cephalgia. Lancet Neurol 1:251-257.

Headache Classification Committee of the International Headache Society (2004) The international classification of headache disorders, Ed 2. Cephalalgia 24:1-160.

Kumar K, Toth C, Nath RK (1997) Deep brain stimulation for intractable pain: a 15-year experience. Neurosurgery 40:736-746; discussion $746-737$.

Leone M, Franzini A, Bussone G (2001) Stereotactic stimulation of posterior hypothalamic gray matter in a patient with intractable cluster headache. N Engl J Med 345:1428-1429.

Leone M, Franzini A, Broggi G, May A, Bussone G (2004a) Long-term follow-up of bilateral hypothalamic stimulation for intractable cluster headache. Brain 127:2259-2264.

Leone M, May A, Franzini A, Broggi G, Dodick D, Rapoport A, Goadsby P, Schoenen J, Bonavita V, Bussone G (2004b) Deep brain stimulation for intractable chronic cluster headache: proposals for patient selection. Cephalalgia 24:934-937.

Leone M, Franzini A, D’Amico D, Grazzi L, Rigamonti A, Mea E, Broggi G, Bussone G (2006) Long-term follow-up of hypothalamic stimulation to relieve intractable chronic cluster headache. Neurology, in press.

Lopez R, Young SL, Cox VC (1991) Analgesia for formalin-induced pain by lateral hypothalamic stimulation. Brain Res 563:1-6.

Malick A, Strassman RM, Burstein R (2000) Trigeminohypothalamic and reticulohypothalamic tract neurons in the upper cervical spinal cord and caudal medulla of the rat. J Neurophysiol 84:2078-2112.

May A (2003) Headache: lessons learned from functional imaging. Br Med Bull 65:223-234. 
May A (2005) Cluster headache: pathogenesis, diagnosis, and management. Lancet 366:843-855.

May A, Bahra A, Büchel C, Frackowiak RSJ, Goadsby PJ (1998) Hypothalamic activation in cluster headache attacks. Lancet 352:275-278.

May A, Ashburner J, Buchel C, McGonigle DJ, Friston KJ, Frackowiak RS, Goadsby PJ (1999) Correlation between structural and functional changes in brain in an idiopathic headache syndrome. Nat Med 5:836-838.

Mazars G, Roge R, Mazars Y (1960) Results of the stimulation of the spinothalamic fasciculus and their bearing on the physiopathology of pain. Rev Prat 103:136-138.

Moore RY (1997) Circadian rhythms: basic neurobiology and clinical applications. Annu Rev Med 48:253-266.

Nandi D, Liu X, Joint C, Stein J, Aziz T (2002) Thalamic field potentials during deep brain stimulation of periventricular gray in chronic pain. Pain 97:47-51.

Overeem S, Vliet JA, Lammers GJ, Zitman FG, Swaab DF, Ferrari MD (2002) The hypothalamus in episodic brain disorders. Lancet Neurol 1:437-444.

Randich A, Gebhart GF (1992) Vagal afferent modulation of nociception. Brain Res Brain Res Rev 17:77-99.
Raskin NH, Hosobuchi Y, Lamb S (1987) Headache may arise from perturbation of brain. Headache 27:416-420.

Sano K, Sekino H, Hashimoto I, Amano K, Sugiyama H (1975) Posteromedial hypothalamotomy in the treatment of intractable pain. Confin Neurol 37:285-290

Schoenen J, Di Clemente L, Vandenheede M, Fumal A, De Pasqua V, Mouchamps M, Remacle JM, de Noordhout AM (2005) Hypothalamic stimulation in chronic cluster headache: a pilot study of efficacy and mode of action. Brain 128:940-947.

Sprenger T, Boecker H, Tolle TR, Bussone G, May A, Leone M (2004) Specific hypothalamic activation during a spontaneous cluster headache attack. Neurology 62:516-517.

Vertes RP, Crane AM (1996) Descending projections of the posterior nucleus of the hypothalamus: Phaseolus vulgaris leucoagglutinin analysis in the rat. J Comp Neurol 374:607-631.

Workman BJ, Lumb BM (1997) Inhibitory effects evoked from the anterior hypothalamus are selective for the nociceptive responses of dorsal horn neurons with high- and low-threshold inputs. J Neurophysiol 77:28312835 . 